\title{
Prevalência de longitudinalidade e fatores associados no cuidado às crianças
}

\author{
Prevalence of longitudinality and associated factors in child' care
}

\author{
Bruna Cristiane Furtado Gomes ${ }^{1}$ \\ Orcid: http://orcid.org/0000-0002-4327-1973 \\ Monica Tabata Heringer Streck ${ }^{3}$ \\ Orcid: http://orcid.org/0000-0002-7906-9785 \\ Jessye Melgarejo do Amaral Giordani ${ }^{5}$ \\ Orcid: http://orcid.org/0000-0002-3825-9734
}

\author{
Gabriele Rissotto Menegazzo ${ }^{2}$ \\ Orcid: http://orcid.org/0000-0002-4181-0267 \\ Jordana Lopes Carvalho ${ }^{4}$ \\ Orcid:http://orcid.org/0000-0002-0985-2766 \\ Teresinha Heck Weiller ${ }^{6}$ \\ Orcid:http://orcid.org/0000-0003-2531-0155
}

\begin{abstract}
Resumo
Introdução: A longitudinalidade é um dos princípios da Atenção Primária à Saúde (APS) estabelecida quando há uma relação de vínculo e confiança entre usuários e profissionais. Objetivo: Analisar a prevalência do atributo longitudinalidade na experiência do familiar e/ou cuidador de crianças associado aos fatores individuais e contextuais nos municípios que compõe a quarta Coordenadoria Regional de Saúde do estado do Rio Grande do Sul, Brasil. Método: Foi realizado um estudo transversal multinível com amostra composta por 1.059 familiares e/ou cuidadores de crianças que acessaram os serviços de Atenção Primária à Saúde. Para coleta de dados utilizou-se questionário de caracterização e o Instrumento de Avaliação da Atenção Primária à Saúde Brasil versão criança. Os dados foram analisados utilizando o programa estatístico STATA 14.0, onde realizou-se uma análise de regressão de Poisson multinível. Resultados: O estudo revelou que municípios de menor porte apresentaram menor prevalência do atributo, e que, quanto maior a cobertura vacinal maior a prevalência. Ainda, familiares/cuidadores que possuem plano de saúde reconhecem os serviços de APS como fonte regular de cuidado. O modelo final não mostrou associação estatisticamente significante com o atributo avaliado. Conclusão: Não houve distinção na prevalência de longitudinalidade entre os diferentes modelos de atenção e isso demostra que há necessidade de aprimorar o processo de trabalho das equipes de Saúde da Família, visto que este modelo deveria estar mais orientado para a longitudinalidade de que o modelo tradicional e misto.
\end{abstract}

Palavras-chave: Atenção Primária à Saúde. Saúde de Criança. Pesquisa sobre serviços de saúde.

\section{Abstract}

Introduction: Longitudinality is a principles of Primary Health Care (PHC) established when there is a bond and trust relationship between users and professionals. Aim: This study aimed to analyze the prevalence of longitudinality attribute in the experience of parents and/or caregivers of children associated with individual and contextual factors in the municipalities

\footnotetext{
${ }^{1}$ Programa de Pós-Graduação em Enfermagem, Universidade Federal de Santa Maria, RS, Brasil. E-mail: brunacfurtado@gmail.com

${ }^{2}$ Programa de Pós-Graduação em Ciências Odontológicas, Universidade Federal de Santa Maria, RS, Brasil. E-mail:

gabi_menegazzo@hotmail.com

${ }^{3}$ Programa de Pós-Graduação em Enfermagem, Universidade Federal de Santa Maria, RS, Brasil. E-mail: monicastreck@hotmail.com

${ }^{4}$ Programa de Pós-Graduação em Enfermagem, Universidade Federal de Santa Maria, RS, Brasil. E-mail: dana.lopes@hotmail.com

${ }^{5}$ Programa de Pós-Graduação em Ciências Odontológicas, Universidade Federal de Santa Maria, RS, Brasil. E-mail: jessyesm@hotmail.com

${ }^{6}$ Programa de Pós-Graduação em Enfermagem, Universidade Federal de Santa Maria, RS, Brasil. E- mail: weiller2@hotmail.com
} 
from fourth Regional Health Coordination of the state of Rio Grande do Sul, Brazil. Methods: This is a multilevel cross-sectional study that was carried out with a sample of 1,059 parents and/or caregivers of children who accessed the Primary Health Care services. For data collection, a characterization questionnaire and the Primary Health Care Assessment Instrument Brazil, child version were used. The data were analyzed using the statistical software STATA 14.0, where a multilevel Poisson regression model was performed. Results: The study shows that smaller municipalities had a lower prevalence of the attribute, and that the greater the vaccination coverage, the greater the prevalence. Also, parents/caregivers who have a health plan recognize PHC services as a regular source of care. The final model did not show a statistically significant association with the evaluated attribute. Conclusion: There was no distinction in the prevalence of longitudinality between the different models of care and this shows that we need to improve the work process of the Family Health teams, since this model should be more oriented towards longitudinality than the traditional and mixed models.

Keywords: Primary health care. Child health. Health services research.

\section{Introdução}

A Atenção Primária à Saúde (APS) constitui o primeiro nível de contato dos usuários com os sistemas de saúde, principal elemento de um continuado processo de saúde, se configurando como estratégia para produzir melhorias sustentáveis e maior equidade no estado de saúde da população. ${ }^{1}$ No Brasil, a APS é representada por três modelos de atenção distintos: as Unidades Básicas de Saúde tradicionais (UBS), as Estratégias de Saúde da Família (ESF) e as Unidades Mistas (UBS e USF).

A APS traz consigo atributos estruturantes considerados essenciais para sua efetividade, sendo eles: o acesso de primeiro contato, longitudinalidade, integralidade, e coordenação do cuidado; e ainda atributos derivados, os quais qualificam as ações na APS: orientação familiar, orientação comunitária e competência cultural. ${ }^{2}$ Dentre eles, destacase o atributo da longitudinalidade, o qual se efetiva quando o usuário reconhece um serviço de saúde como sua fonte habitual de cuidado e o utiliza ao longo do tempo. A longitudinalidade é estabelecida quando há uma relação de vínculo e confiança entre usuários e profissionais, e promove a continuidade de cuidados e informações em saúde. ${ }^{3-4}$

Em relação ao cuidado à criança na APS, o Ministério da Saúde (MS) vem desenvolvendo estratégias como a Política Nacional de Atenção Integral a Saúde da
Criança (PNAISC), que orienta e qualifica ações e serviços de saúde da criança. A PNAISC tem o objetivo de potencializar a qualidade de vida e garantir a longitudinalidade da atenção a partir de mudanças do modelo técnico-assistencial, representada pela ampliação do acesso aos serviços, desfragmentação da assistência e mudança na forma como o cuidado às gestantes e aos recém-nascidos vem sendo realizado. ${ }^{5}$ Desta forma, os cuidados à criança na APS devem iniciar na primeira semana de vida, e seguir de forma continuada, envolvendo condições do contexto de saúde e de vida da família, com articulações intersetoriais, no território, conforme as necessidades de cada criança/família.

A expansão da APS através da ampliação das ESF, no Brasil, contribuiu para a redução da mortalidade infantil, porém, ainda prevalece o cuidado à criança pautado na queixa-conduta, com ações insuficientes para garantir a presença e a extensão da longitudinalidade, pois existe um desequilíbrio entre a oferta do cuidado e as necessidades das crianças, permanecendo a longitudinalidade como desafio para os modelos assistenciais. ${ }^{6-8}$

A avaliação dos serviços de APS permite conhecer sua efetividade e remodelar as práticas em saúde, objetivando o mais alto grau de orientação para esse nível de atenção. Para contribuir com a avaliação, Starfield ${ }^{2}$ e colaboradores desenvolveram o Primary Care Assessment Tool (PCATool) o qual permite mensurar a 
presença e extensão dos atributos da APS através de entrevistas com familiar e/ou cuidadores de crianças. Este capta aspectos de estrutura, processo e resultados nos serviços, exigindo reafirmação ou reformulação na busca da qualidade tanto para o planejamento e execução das ações de APS independente do modelo de atenção. A versão criança do PCATool foi validada em 2002 no Brasil e seus resultados indicam que o instrumento possui adequada validade e confiabilidade. ${ }^{9}$

Ressalta-se então a importância do cuidado longitudinal à criança, pois sabe-se que o mesmo tem influência na qualidade de vida dela e de seus cuidadores, no desenvolvimento da resiliência e autoestima da criança, na formação de relações e autoproteção, além da visão integral e resolutividade dos problemas trazer facilidades para prescrição de ações de prevenção, precisão nos diagnósticos e redução de custos ao sistema público de saúde. ${ }^{8}$ Assim, este estudo tem por objetivo analisar a prevalência do atributo longitudinalidade na experiência do familiar e/ou cuidador de crianças, associada aos fatores individuais e contextuais nos municípios que compõem a $4^{\text {a }}$ Coordenadoria Regional de Saúde do estado do Rio Grande do Sul ( $\left.4^{\mathrm{a}} \mathrm{CRS} / \mathrm{RS}\right)$.

\section{Materiais e Métodos}

\section{Delineamento e amostra do estudo}

Trata-se de um estudo epidemiológico observacional transversal, onde não é realizada nenhuma intervenção, apenas a observação de um recorte temporário da situação em caráter multinível, pois leva em consideração o contexto em que os indivíduos estão alocados. ${ }^{10}$ Este estudo é decorrente do protejo matricial "Primary Care Assessment Tool (PCATool): Avaliação da Atenção Primária na $4^{\mathrm{a}}$ Coordenadoria Regional de saúde do Estado do Rio Grande do Sul (4 CRS/RS)" e foi realizado nos municípios pertencentes à $4^{\mathrm{a}} \mathrm{CRS} / \mathrm{RS} .^{11}$
A pesquisa foi realizada em 148 unidades de APS distribuídas nos 32 municípios pertencentes a $4^{\mathrm{a}} \mathrm{CRS} / \mathrm{RS}$, localizada na região central do Estado do Rio Grande do Sul. Esta é subdividida em duas regiões de saúde denominadas Verdes Campos e Entre Rios, com 542.357 habitantes no ano da coleta de dados. ${ }^{12}$

A região Verdes Campos, com população de 417.906 habitantes e 101 unidades de APS é composta por 21 municípios sendo eles; Santa Maria; Quevedos; Júlio de Castilhos; Pinhal Grande; Toropi; São Martinho da Serra; Nova Palma; São Pedro do Sul; Itaara; Ivorá; Faxinal do Soturno; Dona Francisca; Silveira Martins; São João do Polesine; Agudo; Dilermando Aguiar; Restinga Seca; Paraíso do Sul; Formigueiro; São Sepé e Vila Nova do Sul. A região Entre Rios possui uma população de 123.915 habitantes e 47 unidades de APS, sendo integrada por 11 municípios, a saber: Santiago, Itacurubi, Unistalda, Capão do Cipó, São Francisco de Assis, Esperança do Sul, Jaguari, Jari, Mata, São Vicente do Sul e Cacequi. ${ }^{12}$

O tamanho da amostra foi definido através do cálculo amostral, considerando a estimativa da média populacional de crianças de cada município pertencente a $4^{\mathrm{a}}$ CRS/RS. A amostra final foi composta por 1.061 familiares e/ou cuidadores de crianças que acessam os serviços de APS da $4^{\mathrm{a}}$ CRS/RS. A amostragem foi nãoprobabilística, por conveniência, a qual envolve os participantes convenientes disponíveis para participar do estudo, ou seja, aqueles que se encontravam disponíveis nas Unidades básicas de Saúde nos momentos de coleta. Utilizou-se como critério de inclusão: que o participante deveria ser cuidador ou responsável legal de uma criança e ter acessado mais de uma vez as unidades de APS para obtenção de cuidados em saúde. Definiu-se como critério de exclusão: usuários com dificuldades para responder ao instrumento. 
avaliadas

Coleta de dados e Variáveis

Para a realização da coleta de dados foi utilizado um questionário de caracterização e o Instrumento de Avaliação da Atenção Primária à Saúde PCATool Brasil versão criança. ${ }^{9}$ A coleta de dados ocorreu no período de fevereiro a junho de 2015 e a mesma foi realizada por estudantes de pós-graduação do curso de enfermagem da Universidade Federal de Santa Maria por meio de tablets com o software Epi Info ${ }^{\circledR}$ 7.0. O instrumento foi aplicado de forma presencial, e os dados foram coletados nas Unidades de Saúde, após contato prévio com os coordenadores, em seus horários de funcionamento, de forma a não causar prejuízo no atendimento aos usuários e no trabalho dos profissionais. As avaliações foram realizadas em local reservado, a fim de preservar o anonimato e a privacidade dos entrevistados.

Os dados de nível individual foram extraídos da aplicação do instrumento PCAtool-Brasil versão criança e do questionário socioeconômico $\mathrm{e}$ demográfico, já os dados do nível contextual estão relacionados com as características dos municípios nos quais residiam os usuários entrevistados (porte populacional e cobertura vacinal), sendo essas informações extraídas do Instituto Brasileiro de Geografia e Estatística IBGE e do Departamento de Gestão da Tecnologia da Informação (BI Saúde).

Quanto às variáveis contextuais (nível de município) utilizou-se região de saúde a qual o participante pertencia (Verdes Campos e Entre Rios), o porte populacional do município que residia (50 mil habitantes ou mais/25001 até 50 mil/2001 até $25 \mathrm{mil} / 15001$ até $20 \mathrm{mil} /$ 10001 até $15 \mathrm{mil} / 5001$ até $10 \mathrm{mil} / 4001$ até
$5 \mathrm{mil} / 3001$ até $4 \mathrm{mil} /$ até $3 \mathrm{mil}$ ) e a cobertura vacinal de seu município (até $80,62 \%$ / de $80,63 \%$ até $96,43 \%$ / mais de $96,43 \%$ ).

As variáveis individuais utilizadas foram coletadas através de uma entrevista com o familiar e/ou cuidador e incluíram: sexo da criança (masculino/feminino), faixa etária (até 5 anos/6 a 11 anos/12 a 18 anos), se possuía plano de saúde ( $\operatorname{sim} /$ não), qual era o modelo de atenção em saúde acessado (UBS/USF/Mista) e qual sua renda familiar mensal (Até R\$788,00/ R $\$ 789,00$ a $\mathrm{R} \$ 1.576,00 / \mathrm{R} \$ 1.577,00$ a $\mathrm{R} \$ 2.364,00 /$ $\mathrm{R} \$ 2.365,00$ a $\mathrm{R} \$ 3.152,00 /$ Mais de $\mathrm{R} \$ 3.152,00)$.

O desfecho deste estudo foi a prevalência da longitudinalidade no cuidado à criança na APS. $O$ atributo longitudinalidade na APS foi coletado através do PCAtool-Brasil versão criança e foi categorizado de forma dicotômica com os pontos de corte de $<6,6$ para escore baixo do atributo e >6,6 para escore alto do atributo. Quando a soma de respostas em branco e as com valor atribuído "(9)" atingisse $50 \%$ ou mais do total de itens do componente, esse entrevistado era excluído da análise. No caso desses valores serem inferiores a $50 \%$ do total de itens foi realizada uma transformação do valor "(9)" para o valor "(2)". Essa transformação foi considerada necessária para pontuar negativamente quando houvesse características deste instrumento não reconhecidas pelo entrevistado. ${ }^{2}$

\section{Análise estatística}

Para a explicação das relações entre as variáveis foi elaborado um modelo teórico, organizado segundo as dimensões individuais e contextuais do atributo longitudinalidade (Figura 1). 
Figura 1. Modelo teórico da Longitudinalidade no cuidado à criança na atenção primária à saúde, na experiência do familiar e/ou cuidador, segundo características individuais e contextuais.

Nível Contextual

Nível Individual
Desfecho

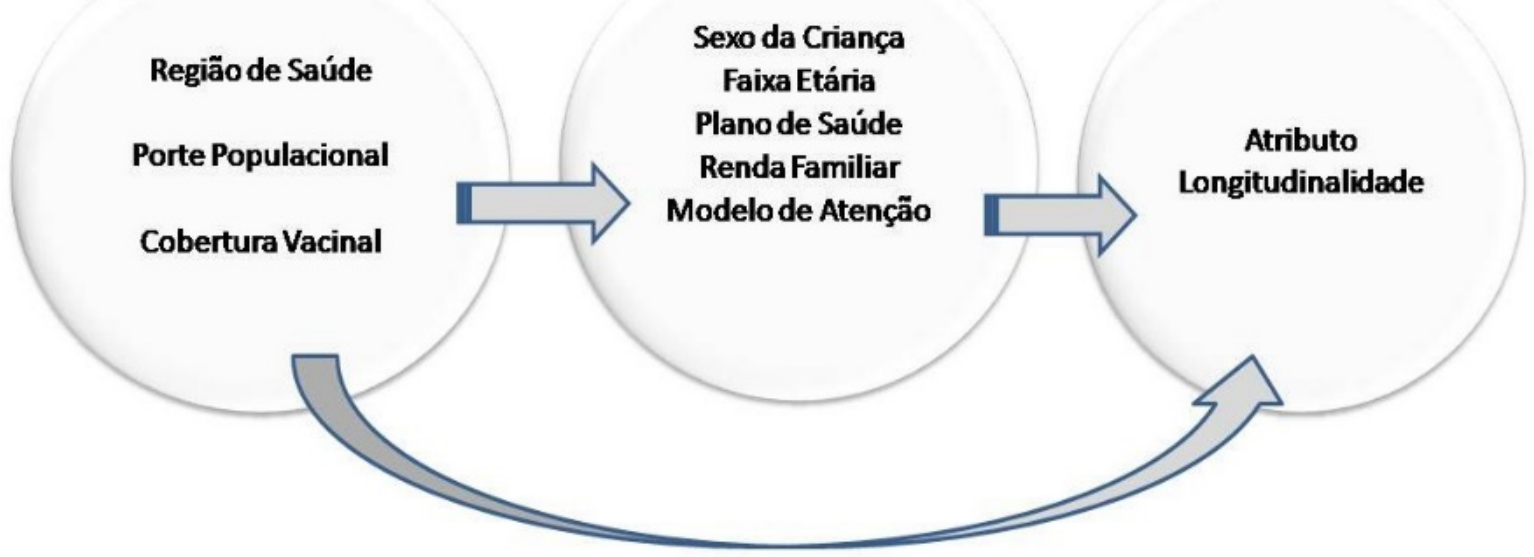

Os dados foram analisados por meio do programa estatístico STATA 14.0 (Stata Corporation, College Station, TX, USA). $\mathrm{Na}$ análise descritiva, verificou-se as frequências absolutas e relativas das variáveis estudadas e a prevalência do atributo avaliado de acordo com cada variável. Foi realizada ainda comparação das proporções das variáveis com o atributo longitudinalidade através do teste Quiquadrado e regressão de Poisson multinível para verificar associação entre as variáveis contextuais e individuais com o atributo, onde no primeiro nível foram considerados os participantes do estudo, incluídos em suas respectivas cidades (segundo nível), para obtenção das razões de prevalência brutas e ajustadas com seus respectivos intervalos de confiança de $95 \%$ e nível de significância de 5\%. A modelagem utilizada ocorreu em dois estágios: Modelo 1 (apenas as variáveis contextuais no ajuste dentro do próprio bloco); e Modelo 2 (as variáveis contextuais mais as variáveis individuais). Para a análise de ajuste dos modelos foi utilizado o deviance (loglikelihood), verificando-se assim alterações estatisticamente significativas entre eles.

\section{Preceitos éticos}

Este estudo é parte de um projeto matricial submetido e aprovado pelo Comitê de Ética de Pesquisa da Universidade Federal de Santa Maria (CAAE 34137314.4.0000.5346).

Quanto aos aspectos éticos do presente estudo, foram assegurados de acordo com a resolução $\mathrm{n}^{\mathrm{0}} 466$ de 12 de dezembro de 2012 que diz respeito a pesquisas envolvendo seres humanos. $\mathrm{O}$ projeto foi financiado com recursos do Conselho Nacional de Desenvolvimento Científico e Tecnológico Edital 447000/2017-4.

\section{Resultados}

Foram considerados no estudo 1.059 respondentes, os quais completaram 50\% ou mais das respostas referentes à longitudinalidade. Os resultados da avaliação da longitudinalidade da APS, de acordo com a experiências do familiar e/ou cuidador de crianças, são apresentados na tabela 1 . 
Tabela 1. Descrição da amostra, prevalência do atributo longitudinalidade e razões de prevalência bruta utilizando regressão de Poisson multinível (n=1.059).

\begin{tabular}{|c|c|c|c|c|}
\hline VARIÁVEL & n (\%) & $\begin{array}{c}\text { Prevalência da } \\
\text { Longitudinalidade } \\
\text { (IC } 95 \%)\end{array}$ & $\begin{array}{c}\text { RP }^{\mathrm{b}} \text { bruta }\left(\mathrm{IC}^{\mathrm{a}}\right. \\
95 \%)\end{array}$ & Valor $\mathbf{P}$ \\
\hline Região & & $0,365^{*}$ & & \\
\hline Verdes campos & $811(76,58)$ & $71,1(67,9-74,1)$ & 1 & \\
\hline Entre Rios & $248(23,42)$ & $68,1(62,06-73,6)$ & $0,95(0,80-1,13)$ & 0,622 \\
\hline Porte Populacional & & $0,260 *$ & & \\
\hline 50 mil ou mais & $499(47,08)$ & $72,1(68,0-75,9)$ & 1 & \\
\hline 25001 até $50 \mathrm{mil}$ & $92(8,68)$ & $70,6(60,5-79,08)$ & $0,97(0,75-1,27)$ & 0,877 \\
\hline 2001 até $25 \mathrm{mil}$ & $110(10,38)$ & $75,4(66,5-82,6)$ & $1,04(0,82-1,32)$ & 0,713 \\
\hline 15001 até $20 \mathrm{mil}$ & $112(10,57)$ & $61,6(52,2-70,1)$ & $0,85(0,66-1,10)$ & 0,230 \\
\hline 10001 até $15 \mathrm{mil}$ & $54(5,9)$ & $77,7(64,6-87,0)$ & $1,07(0,78-1,48)$ & 0,644 \\
\hline 5001 até $10 \mathrm{mil}$ & $93(8,77)$ & $60,2(49,9-69,6)$ & $0,83(0,62-1,10)$ & 0,208 \\
\hline 4001 até 5 mil & $31(2,92)$ & $90,3(73,5-96,9)$ & $1,25(0,85-1,83)$ & 0,252 \\
\hline 3001 até 4 mil & $39(3,68)$ & $53,8(38,1-68,8)$ & $0,74(0,48-1,15)$ & 0,193 \\
\hline Até 3 mil & $30(2,83)$ & $76,6(58,1-88,6)$ & $1,06(0,69-1,61)$ & 0,777 \\
\hline Cobertura Vacinal & & $0,520^{*}$ & & \\
\hline Até $80,62 \%$ & $204(19,25)$ & $68,6(61,9-74,6)$ & 1 & \\
\hline $80,63 \%$ até $96,43 \%$ & $739(69,72)$ & $70,9(67,5-74,0)$ & $1,03(0,85-1,24)$ & 0,732 \\
\hline Mais de $96,43 \%$ & $117(11,04)$ & $70,9(62,0-78,4)$ & $1,03(0,78-1,35)$ & 0,811 \\
\hline Sexo & & $0,488 *$ & & \\
\hline Masculino & $514(48,67)$ & $69,4(65,3-73,2)$ & 1 & \\
\hline Feminino & $542(51,33)$ & $71,4(67,4-75,0)$ & $1,02(0,89-1,18)$ & 0,707 \\
\hline Faixa etária & & $0,560 *$ & & \\
\hline Até 5 anos & $477(45,78)$ & $71,9(67,6-75,7)$ & 1 & \\
\hline 6 a 11 anos & $337(32,34)$ & $70,3(65,2-74,9)$ & $0,97(0,82-1,15)$ & 0,792 \\
\hline 12 a 18 anos & $228(21,88)$ & $70,1(63,8-75,7)$ & $0,97(0,80-1,17)$ & 0,799 \\
\hline Plano de Saúde & & $0,631^{*}$ & & \\
\hline Sim & $185(17,54)$ & $71,8(64,9-77,9)$ & 1 & \\
\hline Não & $870(82,46)$ & $70,1(66,9-73,07)$ & $0,97(0,80-1,17)$ & 0,794 \\
\hline Modelo de Atenção & & $0,201 *$ & & \\
\hline UBS & $439(42,17)$ & $74,9(70,6-78,7)$ & 1 & \\
\hline ESF & $456(43,80)$ & $68,4(63,9-72,5)$ & $0,91(0,78-1,06)$ & 0,249 \\
\hline Mista & $146(14,02)$ & $66,4(58,3-73,6)$ & $0,88(0,70-1,11)$ & 0,297 \\
\hline Renda Familiar & & $0,131^{*}$ & & \\
\hline Até $\mathrm{R} \$ 788,00$ & $417(39,64)$ & $68,1(63,4-72,4)$ & 1 & \\
\hline $\mathrm{R} \$ 789,00$ a $\mathrm{R} \$ 1.576,00$ & $451(42,87)$ & $75,1(70,9-78,9)$ & $1,10(0,94-1,29)$ & 0,220 \\
\hline $\mathrm{R} \$ 1.577,00$ a $\mathrm{R} \$ 2.364,00$ & $110(10,46)$ & $72,7(63,6-80,2)$ & $1,06(0,83-1,36)$ & 0,604 \\
\hline $\mathrm{R} \$ 2.365,00$ a $\mathrm{R} \$ 3.152,00$ & $53(5,04)$ & $56,6(42,9-69,3)$ & $0,83(0,57-1,21)$ & 0,335 \\
\hline Mais de $\mathrm{R} \$ 3.152,00$ & $21(2,00)$ & $52,3(31,3-72,5)$ & $0,76(0,42-1,40)$ & 0,393 \\
\hline
\end{tabular}

Valores abaixo de 1.060 fazem parte de dados faltantes.

${ }^{\mathrm{a}} \mathrm{IC}$ : intervalo de confiança; ${ }^{\mathrm{R} P}$ : razão de prevalência.

*Comparação das variáveis com o atributo longitudinalidade pelo valor de $\mathrm{p}$ do teste do qui-quadrado.

Em relação às variáveis contextuais, a maior proporção da amostra $(76,58 \%)$ pertencia à região Verdes Campos, 499 $(47,08 \%)$ eram residentes em municípios com 50 mil ou mais habitantes e 739 $(69,72 \%)$ apresentaram cobertura vacinal de $80,63 \%$ a $96,43 \%$. Na prevalência bruta da longitudinalidade, esta foi de $71,1 \%$ para a região Verdes Campos e $68,1 \%$ para a Região Entre Rios. O porte populacional que mostrou maior prevalência de longitudinalidade foi o que conta com 4001 a 5 mil habitantes $(90,3 \%)$, e a cobertura vacinal demonstrou prevalência bruta de longitudinalidade semelhante em todas as categorias. A prevalência geral de longitudinalidade foi de $68,1 \%$ (com o mínimo de $62,1 \%$ e o máximo de $73,6 \%$ ).

No que concerne às variáveis individuais, houve distribuição em igual proporção entre o sexo da criança, porém, a faixa etária com maior representatividade 
foi a de até 5 anos, com 477 (45,78\%), seguida, da faixa etária de 6 a 11 anos, com 337 (32,34\%). A maioria dos participantes não possuía plano de saúde $(82,42 \%)$ e 451 $(42,87 \%)$ indicaram ter renda familiar mensal média de dois salários-mínimos. Em referência aos modelos de atenção, 42,17\% da amostra declara-se afiliada à UBS, 456 (43,80\%) estão adscritos à ESF, e 146 $(14,02 \%)$ identificaram como fonte de cuidado as unidades mistas. No que se refere às variáveis individuais na prevalência do atributo, identificou-se maior prevalência bruta da longitudinalidade $(71,9 \%)$ em crianças de até 5 anos de idade. A maior proporção dos participantes $(82,46 \%)$ não possuem plano de saúde e entre os que possuem plano de saúde, a prevalência bruta de longitudinalidade foi semelhante entre as características. Observou-se que, quanto menor a renda familiar, variando de 1 a 3 salários-mínimos, maior a prevalência do atributo avaliado $(68,1 \%$ a 75,1$)$. Em relação ao modelo de atenção, a prevalência bruta da longitudinalidade foi maior na UBS $(74,9 \%)$, seguida pela ESF $(68,4 \%)$ e por unidades mistas $(66,4 \%)$.

As comparações pelo teste Quiquadrado e associações por regressão não ajustada entre as variáveis contextuais e individuais e o atributo de longitudinalidade não são estatisticamente significantes. A Tabela 2, então, apresenta os resultados ajustados da análise de regressão de Poisson multinível, onde é feito o acréscimo do bloco de variáveis contextuais (Modelo 1) na análise e, após, das variáveis individuais de cada criança junto a essas (Modelo 2), mostram-se um modelo mais explicativo da situação.

Tabela 2. Razões de Prevalência ajustadas para as variáveis do nível de equipe de saúde e variáveis individuais utilizando regressão de Poisson multinível $(\mathrm{n}=1.060)$.

\begin{tabular}{|c|c|c|c|c|}
\hline VARIÁVEL & $\begin{array}{c}\text { Modelo 1 } \\
\text { RP }^{\mathbf{a}} \text { ajustada (IC } \\
95 \%)\end{array}$ & Valor P & $\begin{array}{c}\text { Modelo 2 } \\
\text { RP }^{\mathrm{a}} \text { ajustada } \\
(\text { ICb 95\%) } \\
\end{array}$ & Valor $\mathbf{P}$ \\
\hline \multicolumn{5}{|l|}{ Região } \\
\hline Verdes campos & 1 & & 1 & \\
\hline Entre rios & $0,88(0,64-1,20)$ & 0,432 & $0,88(0,63-1,25)$ & 0,500 \\
\hline \multicolumn{5}{|l|}{ Porte populacional } \\
\hline 50 mil ou mais & 1 & & 1 & \\
\hline 25001 até $50 \mathrm{mil}$ & $1,11(0,73-1,68)$ & 0,614 & $1,21(0,77-1,88)$ & 0,394 \\
\hline 2001 até $25 \mathrm{mil}$ & $1,19(0,76-1,85)$ & 0,436 & $1,34(0,82-2,16)$ & 0,231 \\
\hline 15001 até $20 \mathrm{mil}$ & $0,92(0,64-1,31)$ & 0,648 & $1,00(0,68-1,47)$ & 0,982 \\
\hline 10001 até $15 \mathrm{mil}$ & $1,26(0,79-2,01)$ & 0,319 & $1,36(0,83-2,24)$ & 0,215 \\
\hline 5001 até $10 \mathrm{mil}$ & $0,83(0,61-1,14)$ & 0,264 & $0,89(0,62-1,27)$ & 0,535 \\
\hline 4001 até 5 mil & $1,24(0,79-1,93)$ & 0,339 & $1,40(0,86-2,26)$ & 0,166 \\
\hline 3001 até 4 mil & $0,80(0,48-1,33)$ & 0,396 & $0,95(0,56-1,62)$ & 0,876 \\
\hline Até 3 mil & $1,07(0,64-1,81)$ & 0,778 & $1,23(0,71-2,14)$ & 0,446 \\
\hline \multicolumn{5}{|l|}{ Cobertura Vacinal } \\
\hline Até $80,62 \%$ & 1 & & 1 & \\
\hline $80,63 \%$ até $96,43 \%$ & $1,13(0,78-1,64)$ & 0,491 & $1,28(0,86-1,92)$ & 0,220 \\
\hline Mais de $96,43 \%$ & $1,24(0,83-1,84)$ & 0,284 & $1,30(0,85-1,99)$ & 0,216 \\
\hline \multicolumn{5}{|l|}{ Sexo } \\
\hline Masculino & & & 1 & \\
\hline Feminino & & & $1,02(0,88-1,19)$ & 0,716 \\
\hline \multicolumn{5}{|l|}{ Faixa etária } \\
\hline Até 5 anos & & & 1 & \\
\hline 6 a 11 anos & & & $0,96(0,81-1,14)$ & 0,682 \\
\hline 12 a 18 anos & & & $0,98(0,81-1,19)$ & 0,861 \\
\hline \multicolumn{5}{|l|}{ Renda Familiar } \\
\hline Até R7488,00 & & & 1 & \\
\hline $\mathrm{R} \$ 789,00$ a $\mathrm{R} \$ 1.576,00$ & & & $1,05(0,89-1,25)$ & 0,524 \\
\hline $\mathrm{R} \$ 1.577,00$ a $\mathrm{R} \$ 2.364,00$ & & & $1,02(0,78-1,34)$ & 0,851 \\
\hline $\mathrm{R} \$ 2.365,00$ a $\mathrm{R} \$ 3.152,00$ & & & $0,77(0,52-1,15)$ & 0,218 \\
\hline
\end{tabular}




\begin{tabular}{lcc}
\hline Mais de R\$3.152,00 & $0,91(0,47-1,76)$ & 0,795 \\
Plano de Saúde & 1 & \\
Sim & $0,94(0,76-1,17)$ & 0,615 \\
Não & 1 & \\
Modelo de Atenção & 1 & \\
UBS & $0,86(0,71-1,04)$ & 0,139 \\
ESF & $0,84(0,64-1,11)$ & 0,240 \\
\hline
\end{tabular}

Modelo 1: apenas as variáveis contextuais no ajuste dentro do próprio bloco.

Modelo 2: variáveis contextuais com $\mathrm{p}<0,05$ do modelo 2 , mais as variáveis do nível individual de cada criança. ${ }^{\mathrm{a} R P}$ : razão de prevalência; ${ }^{\mathrm{b}} \mathrm{IC}$ : intervalo de confiança.

As razões de prevalência ajustadas para as variáveis do nível contextual e variáveis individuais apresentaram valores de $\mathrm{p}$ superiores a 0,05 . Este resultado demonstra que, tanto o modelo contextual quanto o modelo final, não mostraram associações estatisticamente significantes com o atributo avaliado.

\section{Discussão}

O estudo revelou que não houve diferenças na prevalência de longitudinalidade conforme as variáveis estudadas. Estes achados corroboram os estudos que utilizaram o PCATool versão criança e evidenciaram que uma vez o usuário acesse o serviço de saúde, ele considera o serviço e os profissionais como uma fonte de cuidado para as consultas de rotina e novas demandas de saúde, e se constituem relações de pertencimento, vínculo e confiança. ${ }^{13-16}$

Em relação às variáveis contextuais (nível do município) que compõe o modelo, a maior proporção da amostra residia em municípios com 50 mil ou mais habitantes. Os dados revelaram que a prevalência do atributo longitudinalidade varia de acordo com o porte populacional do município. Isso representa um desafio para a gestão, onde os municípios de menor porte requerem maior suporte técnico da Secretaria Estadual de Saúde e de suas estruturas regionais, bem como a valorização dos Colegiados de Gestão Regionais, responsáveis pela pactuação do fluxo dos usuários na rede de serviços intermunicipais. ${ }^{17} \quad$ Quanto ao acompanhamento da situação vacinal da criança uma das ações programáticas prioritárias das equipes de APS, este estudo revelou que quanto maior a cobertura vacinal, maior a prevalência do atributo longitudinalidade. Depreende-se que há relação entre o vínculo do familiar e/ou cuidador com os serviços de APS e a cobertura vacinal. Entretanto há uma lacuna na produção científica no que tange a relação da situação vacinal com a prevalência da longitudinalidade.

Observou-se maior prevalência do atributo longitudinalidade entre as crianças com até 5 anos, compreendendo que ela frequenta mais os serviços de saúde nos primeiros anos de vida, recebendo orientações de prevenção e promoção de acordo com a faixa etária. Isso corrobora os outros estudos que identificaram que a maior parte dos atendimentos às crianças ocorrem por meio da atenção à demanda espontânea, ocasionados por condições agudas de doenças e agravos e, na maioria das vezes, os responsáveis procuram o primeiro atendimento em unidades de urgência e emergência, descaracterizando a APS como coordenadora da atenção do cuidado. ${ }^{16,18,19}$

A maioria dos participantes não possui plano de saúde e utilizam exclusivamente os serviços de APS. Porém, familiares e/ou cuidadores que possuem plano de saúde reconhecem os serviços de APS como fonte regular de cuidado e identificam a prevalência do atributo longitudinalidade nestes serviços. Dados semelhantes foram identificados em um estudo realizado na Argentina, no qual cuidadores de crianças que utilizavam serviços públicos e privados identificaram a 
maior extensão da longitudinalidade em serviços públicos. ${ }^{20}$ Em estudo realizado no mesmo cenário com usuários adultos, o atributo atingiu escores positivos somente entre aqueles que utilizavam serviços públicos. ${ }^{21}$ Depreende-se que, mesmo utilizando um plano de saúde privado, ainda assim a criança mantém vínculo com os serviços públicos para cuidados de rotina como as imunizações, e acompanhamento do crescimento e desenvolvimento.

Em relação à renda familiar, o estudo demonstrou que familiares e/ou cuidadores que relataram renda de até três salários mínimos faziam uso do serviço público e identificaram a presença do atributo longitudinalidade, o que se difere dos resultados de um estudo realizado na Espanha, no qual familiares e/ou cuidadores de crianças com baixas condições socioeconômicas não identificaram a presença da continuidade do cuidado, visto que diferenças sócio demográficas resultaram em desigualdade na qualidade e provisão do serviço. ${ }^{20}$

Estes achados reafirmam a importância do papel da APS para as populações mais vulneráveis, espaço fundamental para garantir a atenção às crianças. Entretanto deve-se superar o conceito de atenção primária seletiva, direcionadas para populações pobres, expandindo-a para toda a população, garantindo a universalidade da atenção de forma que todos possam ter acesso à oferta de serviços de excelência de forma que a população não se sujeite a gastos com seguros privados de saúde. ${ }^{17}$

A maior prevalência da longitudinalidade foi identificada nas UBS's tradicionais, seguidas pelas ESF e Unidades Mistas, demonstrando que não foi identificado por usuários diferenças em relação ao processo de trabalho das equipes existente entre os modelos de atenção estudados, divergindo dos achados encontrados em estudo semelhante realizado na mesma região com usuários adultos, na qual a longitudinalidade foi identificada com maior escore $(6,9)$ em ESF. $^{21}$

Outros estudos que utilizaram o PCATool versão criança também identificaram que não há diferença entre os modelos de atenção e no processo de trabalho das equipes. ${ }^{22-24}$ Estes achados podem estar relacionados ao maior acesso da criança na demanda espontânea com casos agudos em curso, a dificuldade de formar vínculo com o familiar e/ou cuidador, a falta de adesão à puericultura e a falta de competência técnica para $o$ acompanhamento da criança. ${ }^{21,22}$ Também pode-se inferir que os usuários têm dificuldade em reconhecer os diferentes modelos de atenção, funcionalidades e compreender a lógica do processo de trabalho das equipes, possivelmente decorrentes da baixa capacidade de comunicação entre equipes e usuários. Assim, manter dois modelos de atenção (UBS e ESF) de forma concorrentes pode contribuir para a fragmentação do cuidado, e para a não superação do modelo biomédico. ${ }^{24}$ Há necessidade de mudanças profundas no processo de trabalho das unidades de saúde com ESF, com o intuito de melhorar a qualidade da atenção e promover efetiva implementação das diretrizes propostas pelo modelo ESF. ${ }^{21}$

Em relação aos achados não significativos, pode haver uma relação da forma não probabilística com a qual a amostra foi coletada, o que pode ter gerado um viés de seleção levando a não diferença estatística entre os indivíduos. Por tratar-se de uma amostra regional, para a seleção da diferença do atributo longitudinalidade em crianças um maior número de indivíduos deveria ser entrevistado, porém o estudo demonstrou a presença do atributo em uma região de saúde, fato que havia se limitado até então a um ou dois municípios, e resultam em uma possibilidade de subsidiar teoricamente gestores para incluir como ponto relevante a longitudinalidade no planejamento de suas ações, proporcionando aos profissionais de saúde uma reflexão sobre os serviços disponíveis 
e prestados à esta população.

Este estudo possui algumas limitações, dentre elas a seleção da amostra que ocorreu de forma não aleatória, sendo composta pelos familiares e/ou cuidadores mais acessíveis, e estes foram entrevistados na unidade de saúde, o que pode ter levado os participantes a emitir parecer positivo, para evitar tensões e possíveis prejuízos na assistência a ser recebida. Ainda em relação aos respondentes, estes possuem dificuldade em compreender os diferentes modelos de atenção e seus fluxos de atendimentos.

O PCATool permite captar a autopercepção e, de forma geral, a percepção do usuário é subjetiva e varia conforme o entendimento e as experiências de cuidado. Por tratar-se de um delineamento transversal, este estudo não possibilita afirmativas de causalidade, apenas associação. Ainda, o estudo foi realizado em uma região de saúde do estado do Rio Grande do Sul, sendo que a maior proporção da amostra estratificada pertencia ao município de maior porte populacional em comparação aos demais, o que pode ter influenciado nos resultados, visto o destaque do município de Santa Maria na região. Porém, A análise multinível é apropriada para lidar com esses dados, pois leva em consideração a variância associada a cada nível, indivíduo e contexto. ${ }^{10}$

\section{Conclusão}

Este estudo analisou a prevalência do atributo longitudinalidade na experiência do familiar e/ou cuidador de crianças pertencentes aos municípios que compõem a $4^{\mathrm{a} C R S}$. Os resultados identificaram que não houve distinção na prevalência de longitudinalidade entre as variáveis avaliadas, incluindo os diferentes modelos de atenção. Demostra-se assim, que há necessidade de aprimorar o processo de trabalho das equipes de Saúde da Família, visto que este modelo deveria estar mais orientado para a longitudinalidade de que o modelo tradicional e misto.

Pode se inferir com base neste inquérito que as ações de APS nos municípios da $4^{\text {a }} \mathrm{CRS} / \mathrm{RS}$ são ofertados de modo a contemplar a longitudinalidade. Contudo, há necessidade de realizar diferentes tipos de estudos, avançando para a avaliação de $4^{\text {a }}$ geração, captar os processos de trabalho, a organização e a gestão das equipes, bem como a relação de vínculo e comunicação com o usuário, para assim refletir sobre parâmetros de qualidade e extensão dos serviços fazendo valer os princípios que norteiam a APS.

Este estudo traz contribuições para a enfermagem, considerando que esta categoria profissional tem papel preponderante nas equipes de APS, na gestão e coordenação das equipes, assegurando ou não os atributos essenciais, dentre eles, a longitudinalidade da atenção. Em relação ao cuidado da criança, o estudo sinaliza a importância da APS ser a ordenadora do cuidado da criança, aprimorando o vínculo, e comunicação entre profissionais de saúde e usuários, e profissionais da APS com serviços especializados.

\section{Referências}

1. Organização Pan-Americana da Saúde. Renovación de la Atención Primaria de Salud en las Américas. Brasil: Organização Panamericana de Saúde; 2005.

2. Brasil. Ministério da Saúde. Secretaria de Atenção em Saúde. Departamento de Atenção Básica. Manual do instrumento de avaliação da atenção primária à saúde: primary care assessment tool PACTool - Brasil. Brasília: Ministério da Saúde; 2010. 
3. Cunha EM, Giovanella L. Longitudinalidade/Continuidade do Cuidado: identificando dimensões e variáveis para a avaliação da Atenção Primária no contexto do sistema público de saúde brasileiro. Ciências e saúde Coletiva 2011; 16(1):1029-1042.

4. Starfield B. Atenção primária: equilíbrio entre necessidades de saúde, serviços e tecnologia. Brasília: UNESCO, Ministério da Saúde; 2002.

5. Brasil. Ministério da Saúde. Secretaria de Atenção à Saúde. Departamento de Atenção Básica. Saúde da criança: crescimento e desenvolvimento. Brasília: Ministério da Saúde; 2012. [Cadernos de Atenção Básica, no 33]

6. Morais JMO, Nogueira CMCS, Morais FRR. First contact access in primary health care for children from 0 to 9 years old. Rev Cuidado Fundamental. 2017; 9(3):848-856.

7. Silva AS, Fracolli LA. Evaluation of child care in the Family Health Strategy. Rev. Bras. de Enfermagem. 2016; 69(1):54-61.

8. Oliveira VC, Verissimo MOR. The practice of longitudinality in attendance of children's health: comparison between distinct care models. Rev. Cogitare Enfermagem 2015; 20(1):45-52.

9. Harzheim E, Starfield B, Rajmil L, Dardet CA, Stein AT. Consistência interna e confiabilidade da versão em português do Instrumento de Avaliação da Atenção Primária (PCATool-Brasil) para serviços de saúde infantil. Cad. Saúde Pública 2006; 22(8):1649-1659.

10. Diez-Roux AV. Multilevel analysis in public health research. Annu Rev Public Health 2000; 21:171-92.

11. Damaceno NA, Bandeira $D$, Weiller TH. Avaliação do acesso de primeiro contato na atenção primária da $4^{a}$ Coordenadoria Regional de Saúde do Estado do Rio Grande do Sul. Biblioteca Lascasas, 2016; 12(2).

12. Instituto Brasileiro de Geografia e Estatística. Diretoria de Pesquisa - DPE. Coordenação de População e Indicadores Sociais - COPIS. Nota: Estimativa da população residente com data de referencia $1^{\circ}$ de julho de 2011.

13. Wolkers PCB, Macedo JCB, Rodrigues CM, Furtado MCC, Mello DF. Primary care for children with type 1 diabetes mellitus: perspective of caregivers. Rev.Acta paulista enfermagem 2017; 30(5):451-457.

14. Silva CB, Paula CC, Lopes LFD, Harzheinm E, Magnago TSBS, Schimith MD. Health care for children and adolescents with HIV: a comparison of services. Rev.Brasileira de Enfermagem 2016; 69(3):522-531.

15. Harzheim E, Pinto LF, Hauser L, Soranz D. Assessment of child and adult users of the degree of orientation of Primary Healthcare in the city of Rio de Janeiro, Brazil. Rev. Ciências e Saúde Coletiva 2016; 21(5):1399-1408.

16. Fracolli LA, Muramatsu MJ, Gomes MFP, Nabão FRZ. Evaluation of the Primary Healthcare attributes in a municipality located in the countryside of São Paulo State Brazil. Rev. o mundo da Saúde 2015; 15(39):54-61. 
17. Brasil. Conselho Nacional de Secretários Estaduais de Saúde. Organização PanAmericana da Saúde inovando o papel da Atenção primária nas redes de Atenção à Saúde: resultados do laboratório de inovação em quatro capitais brasileiras. Brasília: Organização Pan Americana da saúde; 2011.

18. Streck MTH, Gomes BCF, Carvalho JL. Soder AB, Weiller TH, Damaceno AN. First contact access of the child to primary health care services: integrative review. International Journal Of Development Research 2018; 8(10):23315-23321.

19. Finkler AL,Viera CS, Tacla MT, Toso BR . O acesso e a dificuldade na resolutividade do cuidado da criança na atenção primária à saúde. Rev. Acta Paulista de Enfermagem 2014; 27(6):548-5.

20. Rivera $\mathrm{C}$, Felló G, Berra S. La experiencia de continuidad con el proveedor de atención primaria en escolares. Rev Bras Med Fam Comunidade 2013; 8(29):227-34.

21. Kessler M, Lima SBS, Weiller TH, Lopes LFD, Ferraz L, Thumé E. Longitudinality in Primary Health Care: a comparison between care models. Rev Bras Enferm. 2018; 71(3):1063-71.

22. Frank BRB; Viera CS; Ross C; Obregón PL; Toso BRGO. Avaliação da longitudinalidade em unidades de Atenção Primária à Saúde. Rev. Saúde e Debate 2015; 39(105):400-410.

23. Reichert APS, Rodrigues PF, Albuquerque TM, Collet N, Minayo MCS. Vínculo entre enfermeiros e mães de crianças menores de dois anos: percepção de enfermeiros. Rev. Ciências e Saúde Coletiva 2016; 21(8):2375-2382.

24. Silva AS, Baitelo TC, Fracolli LA. Avaliação da Atenção Primária à Saúde: a visão de usuários e profissionais sobre a Estratégia de Saúde da Família. Rev. Latino Americana de Enfermagem 2015; 15(23):979-87.

\section{Como citar este artigo:}

Gomes BCF, Menegazzo GR, Streck MTH, Carvalho JL, Giordani JMA, Weiller TH. Prevalência de longitudinalidade e fatores associados no cuidado às crianças. Rev. Aten. Saúde. 2021; 19(68): 65-76. 